\title{
Synthesis of 2S,5S-and 2R,5S-5-Hydroxypipecolic Acid via Amide-Methylenation of S-5-Hydroxy-2-piperidone with Dimethyltitanocene.
}

\author{
Claus Herdeis* and Eberhard Heller \\ Inatitut fur Pharmazie und Lebenamittelchemia der \\ Universittat 8700 Worzburg. Am Hublend, FRG
}

(Received in UK 9 July 1993)

\section{Abstract:}

A route to $2 \mathrm{~S}, 5 \mathrm{~S}$-and $2 \mathrm{R}, 5 \mathrm{~S}$-hydroxypipecolic acid is presented, starting with the enantiopure $5 \mathrm{~S}$ 5-hydroxy-piperidone 7. The key step of this reaction sequence is the chemoselective methylenation of the amide carbonyl group of 8 with dimethyltitanocene 9 to 10 . The transformation of the exocyclic enecarbamate double bond to the carboxylic acid group is best accomplished via hydroboration/oxidation to the alcohol 11a,b. Separation and oxidation of the diastereomers 11a,b, to 14a, and 14b, and hydrolysis furnishes the diastereomeric pipecolic acids $15 a$ and $15 b$ in enantiopure form.

Substituted pipecolic acids are the subject of much current investigation 1 , especially in the field of medicinal Chemistry ${ }^{2}$. Phosphonic amino acids are an important class of competitive $\mathrm{N}$-methyl-D-aspartate (NMDA) antagonists. So CGS 19755 (1) is a potent NMDA antagonist and a derivative of pipecolic acid. ${ }^{3}$ Replacement of the phosphonic acid functional group by the tetrazole moiety gave the NMDA antagonist 2.4<smiles>O=C(O)C[C@@H]1CCNC[C@@H]1O</smiles>

1<smiles>O=C(O)[C@@H]1CC[C@@H](O)CN1</smiles>

3<smiles>O=C(O)[C@H]1CC[C@@H](Cl)CN1</smiles>

4<smiles>O=C[C@H]1C[C@H](Cc2nnn[nH]2)CCN1</smiles>

2<smiles>N[C@@H](C(=O)O)[C@H]1CC[C@@H](Cl)CN1</smiles><smiles>O=C(O)[C@H]1CC=CCN1</smiles>

6 
Previously we synthesized 3, via a homochiral acyliminium ion 19 and (2R,5R)-5chloropipecolic acid 45 , the first member of a class of enantiopure halogenated pipecolic acids. This compound should have interesting pharmacological properties comparable to streptolutin 5, a compound which was isolated in dimeric form from streptomyces griseolutus 6 . In recent years Baikiain 76 served as a starting material for the synthesis of pipecolic acids, substituted in 4-and 6-positions.

Here we report an alternative method leading to cis and trans 5-OH-pipecolic acid (15a,b) with predominant formation of the cis diastereomer (2S,5S)-15a. In conjunction with our work on the synthesis of 3 and derivatives thereof starting from 8 (derived from 7), we investigated the reactivity of the amide group of 8 towards various organometallic reagents. We have found that the highly electrophilic, carbamate activated amide carbonyl group of 8 , is readily attacked by metallorganic reagents (e.g. organocuprates) affording ring open products. With the Grubbs-Tebbe reagent ${ }^{8}$ we isolated the exocyclic enecarbamate 10 in $40-50 \%$ yield.<smiles>O=C1CC[C@@H](O)CN1</smiles>

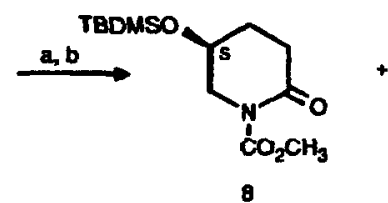

[8] - TBOMS<smiles>C[Te](C)([O-])[Hg]</smiles>

9

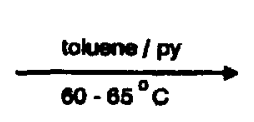<smiles>C=C1CC[C@@H]([Hg])CN1C(=O)O</smiles><smiles>COCCO</smiles>

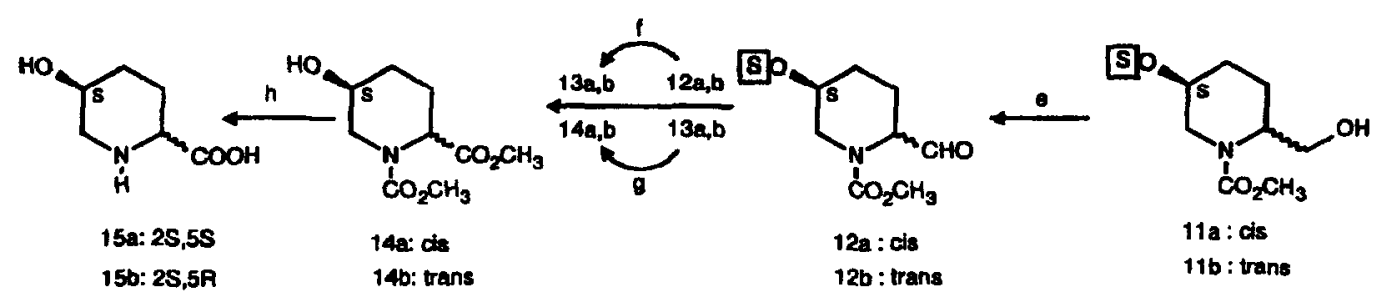

a: TBDMSCl, imidazole, DME; b: BuLi, $\mathrm{CNCO}_{2} \mathrm{CH}_{3}$, THF; c: 9-BBN; d: $3 \mathrm{M} \mathrm{NaOH} / \mathrm{H}_{2} \mathrm{O}_{2}$;

e: Swern-Ox.; I: $\mathrm{Br}_{2} / \mathrm{MeOH} / \mathrm{NaHCO}_{3} ; \mathrm{g}$ : $\mathrm{HCl} / \mathrm{MeOH} ; \mathrm{h}: 6 \mathrm{M} \mathrm{HCl} / \mathrm{\Delta}$

With the Oshima-Lombardo reagent $\left(\mathrm{Zn}-\mathrm{CH}_{2} \mathrm{Br}_{2}-\mathrm{TiCl}_{4}\right)^{9}$ decomposition of the starting material 8 occurred. It turned out subsequently that the inexpensive and easy to handle dimethyltitanocene $9^{10}$ reacted with 8 to give the enecarbamate 10 in $80-85 \%$ yield. Petasis and Bzowej ${ }^{11}$ showed that 9 is an excellent methylenation reagent for aldehydes, ketones, lactones and esters. Methylenation of amides or imides have not been described until now with this reagent 12 . This olefination works also well with other hydroxy protecting groups and 
with the t-butoxycarbonyl (Boc) group on the amide nitrogen of 2-piperidone derivatives 13. Of importance is that a carbamate group is used for protection and activation of the amide moiety. We were not successful in isolating exocyclic $\mathrm{N}$-benzylenamines. In the presence of Lewis acids, 10 is quantitatively transformed to the endocyclic enecarbamate $10 \mathrm{a} .15$

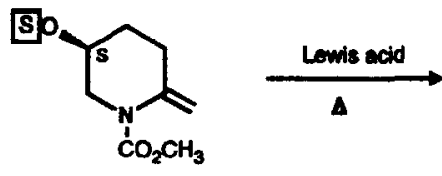

10<smiles></smiles>

$10 \mathrm{a}$

Hydroboration of 10 with $\mathrm{BH}_{3} \cdot \mathrm{S}\left(\mathrm{CH}_{3}\right)_{2}$ and oxidation with $\mathrm{H}_{2} \mathrm{O}_{2} / \mathrm{NaOH}$ furnished $11 \mathrm{a}, \mathrm{b}$ in a cis/trans ratio of - 1:1. With the sterically more demanding 9-BBN the cis/trans ratio was 7:3. The separation of the main portion of the cis diastereomer by flash chromatography was accomplished on this stage.

Direct oxidation of the alcohols $11 \mathrm{a}, \mathrm{b}$ to the carboxylic acids with $\mathrm{RuCl}_{3} / \mathrm{NaIO}_{4}{ }^{16}$ proved to be unsuccessful. However a two step procedure provided $14 \mathrm{a}$ in about $55 \%$ yield. In the first step Swern oxidation of 11 a fumished the carboxaldehyde $12 \mathrm{a}$ which was oxidized with bromine in methanol 17 to the methyl carboxylate 13a. Deprotection of the TBDMS ether was accomplished nearly quantitatively with methanolic hydrogen chloride solution at $0^{\circ} \mathrm{C}$. The resulting $\mathrm{TBDMSOCH}_{3}$ ether was evaporated in vacuo. The subsequent hydrolysis of $14 \mathrm{a}$ with $6 \mathrm{M}$ hydrochloric acid provided 2S,5S-5-hydroxypipecolic acid hydrochloride 15a, identical in all spectroscopic data with material, isolated previously from morus alba 19. On the other hand a complete separation of the diastereomers was possible with $14 a, b$, though more tedious than with $11 \mathrm{a}, \mathrm{b}$. Compound $14 \mathrm{~b}$ was hydrolysed to $15 \mathrm{~b}$, which was identical in every respect with material prepared via the acyl iminium ion pathway $\mathbf{1 g}$. In order to compare the ${ }^{1} \mathbf{H}$-and ${ }^{13} \mathrm{C}-\mathrm{NMR}$ data of $15 \mathrm{a}$, we prepared $(2 \mathrm{R}, 5 \mathrm{R})$-5-hydroxypipecolic acid hydrochloride 18 (ent$15 \mathrm{a}$ ) in an independent way, though only in a cis/trans ratio of 92:8. Starting with 1619, previously prepared by us from (S)-5-hydroxy-2-piperidone, Mitsunobu reaction yielded 17 with complete inversion of configuration in position 5 . Hydrolysis and recrystallisation furnished 18 in $84 \%$ diastereomeric excess.

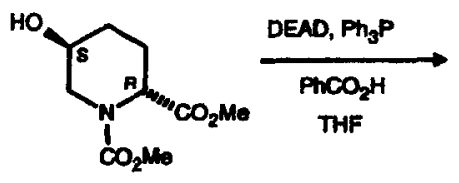

$1680 \%$ d.e.<smiles></smiles>

$1780 \%$ d.e.<smiles>OC1CC[C@@H](O)[C@H]1O</smiles>

18 84\% d.e. 
In summary, the olefination of an activated amide carbonyl group by dimethyl titanocene to exocyclic enecarbamates provides after hydroboration, oxidation and functional group transformations facile access to (2S,5S)-5-hydroxy-pipecolic acid, a compound, which was isolated recently from morus alba. Methylenation reactions with more complex starting materials are currently underway. 13

Acknowledgement. Financial support was provided by Fonds der Chemischen Industrie. We thank Degussa AG Hanau for D and L-glutamic acid and Mrs. A. Betz, University of Würzburg, for preparation of starting materials.

\section{Experimental:}

General:

Diethylether was dried with anhydrous calcium chloride over night, filtered and then distilled over sodium wire under nitrogen. Toluene was distilled over calcium hydride. THF was dried with potassium hydroxide over night, filtrated and distilled over sodium-potassium alloy under nitrogen. Reactions with organometallic and borane compounds were run in flame-dried glassware under dry and oxygen free nitrogen. Thin layer chromatography: Merck precoated silica gel $60 \mathrm{~F}_{254}$ plates. Reaction compounds were visualized by ultraviolet light or by iodine vapour. Melting points: Büchi 510 apparatus, values uncorrected. $[\alpha]_{D}^{20}$ : Perkin-Elmer 241 polarimeter. IR-spectra: Perkin-Elmer 681 infrared spectrometer. ${ }^{1}$ H-NMR and ${ }^{13}$ C-NMR: Bruker AC 200 and Bruker AC 400. Mase spectra: CH7 Varian-MAT (70 eV).

(5S)-5-Hydroxy-2-piperidinone

This compound was prepared in five steps, starting from L-glutamic acid as described in ref. 18.

(5S)-5-t-Butyldimethyleiloxyl-2-piperidinone

This compound was prepared from 1 as described in ref.1g.

(5S)-5-t-Butyldimethylsilyloxy-N-methoxycarbonyl-2-plperidinone (8)

(5S)-5-t-Butyldimethylailyloxyl-2-plperidinone (1.15 g, $5.0 \mathrm{mmol})$ was dissolved in THF (25 ml) and cooled to $-70^{\circ} \mathrm{C}$. Then a $2.0 \mathrm{M}$ solution $\mathrm{n}$-BuLi in cyclohexane $(2.6 \mathrm{ml}, 5.2 \mathrm{mmol})$ was added. After cooling again to $-70^{\circ} \mathrm{C}$ methyl cyanoformate $(0.44 \mathrm{ml}, 5.5 \mathrm{mmol})$ in THF $(15 \mathrm{ml})$ was added. After stirring for $10 \mathrm{~min}$ at this temperature the solution was allowed to warm to $-30^{\circ} \mathrm{C}$. After addition of water $(10 \mathrm{ml})$ and sat. $\mathrm{NaHCO}_{3} / \mathrm{Na}_{2} \mathrm{CO}_{3}$ solution $1: 1(10 \mathrm{ml})$ the organic layer was separated and the aqueous layer was extracted with ethyl acetate $(3 \times 25$ ml). The combined organic layers were dried with anhydrous sodium sulphate. After filtration and evaporation of the solvent the residue was recrystallized from n-pentane. Yield: $1.20 \mathrm{~g}$ 
(83\%) colourless powder.- $R_{f}: 0.46\left(\mathrm{CHCl}_{3} / \mathrm{EtOAC} 9: 1\right)$. $\mathrm{mp} .: 68^{\circ} \mathrm{C} .-[\alpha]_{\mathrm{D}}^{20}=+10.9$ (c $=$ 1.0, МeOH).- IR (KBr): 2960, 2930, 2900, 2850 (CH), 1715, 1705 (C=0), 1285, (C-O) $\mathrm{cm}^{-}$ 1.- ${ }^{1} \mathrm{H}-\mathrm{NMR}\left(\mathrm{CDCl}_{3}\right): \delta(\mathrm{ppm})=0.07\left(6 \mathrm{H}, \mathrm{s}, \mathrm{Me}_{2} \mathrm{Si}\right), 0.88(9 \mathrm{H}, \mathrm{s}, \mathrm{tBuSi}), 1.78-.2 .05(2 \mathrm{H}$, $m, 4-H), 2.38-2.56\left(1 \mathrm{H}, \mathrm{m}, 3-\mathrm{H}_{\mathrm{a}}\right), 2.69-2.88\left(1 \mathrm{H}, \mathrm{m}, 3-\mathrm{H}_{b}\right), 3.68-3.84(2 \mathrm{H}, \mathrm{m}, 6-\mathrm{H})$, $3.87\left(3 \mathrm{H}, \mathrm{s}, \mathrm{OCH}_{3}\right.$ ), $4.26(1 \mathrm{H}, \mathrm{q}, \mathrm{J}=6.0 \mathrm{~Hz}, 5-\mathrm{H})$. $-13 \mathrm{C}-\mathrm{NMR}\left(\mathrm{CDCl}_{3}\right): \delta(\mathrm{ppm})=-4.9$ $\left(\mathrm{SiCH}_{3}\right), 17.9\left(\mathrm{Me}_{3} \mathrm{CSi}\right), 25.6\left(\left(\mathrm{H}_{3} \mathrm{C}\right)_{3} \mathrm{CSi}\right), 28.8(\mathrm{C}-4), 30.8(\mathrm{C}-3), 52.8\left(\mathrm{OCH}_{3}\right), 53.8(\mathrm{C}-6)$, 64.1 (C-5), 154.9 (C=0, urethane), 170.7 (C-2).- $\mathrm{C}_{13} \mathrm{H}_{25} \mathrm{NO}_{4} \mathrm{Si}$ (287.43).- Calcd.: C 54.32 H 8.77 N 4.87 found: C 54.55 H 9.02 N 4.78.

(5S)-5-t-Butyldimethylsilyloxy-N-methoxycarbonyl-2-methylene-piperidine $(10)$

$8(2.5 \mathrm{~g}, 8.7 \mathrm{mmol})$ was dissolved in a mixture of toluene $(75 \mathrm{ml})$ and dry pyridine $(0.75 \mathrm{ml})$. Dimethyltitanocene ${ }^{3}$ (9) $(1.94 \mathrm{~g}, 9.3 \mathrm{mmol})$ was added and the mixture was heated for $18 \mathrm{~h}$ to $60^{\circ} \mathrm{C}$. After this time the tlc test (petroleum ether/EtOAc 2:1) showed almost complete absence of starting material. After evaporation of the solvent the dark brown oil was diluted with pentane $(75 \mathrm{ml})$ and the solution was filtered. The solvent was evaporated and the light brown oil was purified by flash chromatography on silica gel (petroleum ether/EtOAc 2:1, 0.5 \% Et ${ }_{3} N$ ). Yield: $2.0 \mathrm{~g}(82 \%)$ yellow oil.- $R_{f}: 0.72$ (petroleum ether/EtOAc 2:1).- bp.: 99 $101^{\circ} \mathrm{C} / 10^{-2}$ mbar.- IR (neat): 3105, 2960, 2930, $2860(\mathrm{CH}), 1715(\mathrm{C}=0), 1660(\mathrm{C}=\mathrm{C}) \mathrm{cm}^{-1}$ ${ }^{1} \mathrm{H}$-NMR $\left(\mathrm{C}_{6} \mathrm{D}_{6}\right): \delta(\mathrm{ppm})=0.02,0.04(6 \mathrm{H}, 2 \mathrm{~s}, \mathrm{SiMe})_{2}, 0.94(9 \mathrm{H}, \mathrm{s}, \mathrm{t}-\mathrm{BuSi}),, 1.40-1.55(2$ $H, m, 4-H), 1.81-1.90\left(1 \mathrm{H}, \mathrm{m}, 3-\mathrm{H}_{\mathrm{a}}\right), 2.20-2.30\left(1 \mathrm{H}, \mathrm{m}, 3-\mathrm{H}_{\mathrm{b}}\right), 3.33-3.46(1 \mathrm{H}, \mathrm{m}, 6-$ $\mathrm{H}_{\mathrm{a}}$ ) $3.49\left(3 \mathrm{H}, \mathrm{s}, \mathrm{OCH}_{3}\right), 3.51-3.61(1 \mathrm{H}, \mathrm{m}, 5-\mathrm{H}), 3.68-3.76\left(1 \mathrm{H}, \mathrm{m}, 6-\mathrm{H}_{\mathrm{b}}\right), 4.78(1 \mathrm{H}$, $\left.\mathrm{s},=\mathrm{CH}_{\mathrm{E}}\right), 4.96\left(1 \mathrm{H}, \mathrm{s},=\mathrm{CH}_{\mathrm{Z}}\right)$ ). ${ }^{13} \mathrm{C}-\mathrm{NMR}\left(\mathrm{C}_{6} \mathrm{D}_{6}\right) ; \delta(\mathrm{ppm})=-4.8\left(\mathrm{SiCH}_{3}\right), 20.5(\mathrm{Me} 3 \mathrm{CSi})$, $25.9\left(\left(\mathrm{H}_{3} \mathrm{C}_{3} \mathrm{CSi}\right), 30.0\right.$ (C-3), $34.4(\mathrm{C}-4), 52.4(\mathrm{C}-6), 52.5\left(\mathrm{OCH}_{3}\right), 66.4(\mathrm{C}-5), 107.2$ $\left(=\mathrm{CH}_{2}\right), 143.8(\mathrm{C}-2), 155.3(\mathrm{C}=0) .-\mathrm{MS}: \mathrm{m} / \mathrm{z}(\%)=285(1.1 \%)\left[\mathrm{M}^{+}\right], 228(35.1)\left(\mathrm{M}^{+}\right.$. $\mathrm{C}_{3} \mathrm{H}_{7} \mathrm{~N}$ ], 196 (11) [M+-89), 96 (18.5), 94 (35.4), 89 (100).- $\mathrm{C}_{14} \mathrm{H}_{27} \mathrm{NO}_{3} \mathrm{Si}$ (285.43) Calcd.: C 58.91 H 9.54 N 4.91 found.: C 59.23 H 9.76 N 4.87.

\section{(2R,S-5S)-5-t-Butyldimethylailyloxy-N-methoxycarbonyl-2-hydroxymethyl-piperidine $|11 \mathrm{a}, \mathrm{b}|$}

To the enecarbamate $10(1.4 \mathrm{~g}, 4.9 \mathrm{mmol})$ in THF (60 ml) a solution of $0.5 \mathrm{M}$ 9-BBN (10.4 mmol, $5.2 \mathrm{ml}$ ) at $0^{\circ} \mathrm{C}$ was added. The solution was stirred at $25^{\circ} \mathrm{C}$ for $18 \mathrm{~h}$ then $3.0 \mathrm{M}$ sodium hydroxide solution (50 $\mathrm{ml})$ and $30 \%$ hydrogen peroxide $(50 \mathrm{ml})$ were added and stirred for another $2 \mathrm{~h}$. The layers were separated, the aqueous layer was saturated with solid potassium carbonate and extracted with ethyl acetate $(3 \times 70 \mathrm{ml})$. The combined organic layers were dried over anhydrous potassium carbonate, filtered and the solvent was evaporated. The remaining faint yellow oil was diluted with pentane, stored for $2 \mathrm{~h}$ at $4^{\circ} \mathrm{C}$, filtered and the solvent was removed in vacuo. The oily residue was purified by flash chromatography, to give a nearly colourless oil. Yield: $1.31 \mathrm{~g}(88 \%)$ as a mixture of diastereomers in a ratio of 7:3 (cis/trans).- $\mathbf{R}_{f}=0.61$ (EtOAC).- IR (neat): 3440 (OH), 2920 , 2910, $2850(\mathrm{CH}), 1700(\mathrm{C}=0), 1250(\mathrm{C}-\mathrm{O}) \mathrm{cm}^{-1}$.- ${ }^{1} \mathrm{H}-\mathrm{NMR}\left(\mathrm{CDCl}_{3}\right): \delta(\mathrm{ppm})=0.03-0.04$ (6 H, 2s, SiMe2), 0.83 and 0.84 (9 H, s, tBuSi), $1.38-1.77$ (4 H, m, 3-H, 4-H), $2.45-2.75$ (1 $\mathrm{H}$, broad, $\mathrm{OH}), 2.98\left(1 \mathrm{H}, \mathrm{dd}, 6-\mathrm{H}_{\mathrm{g}}, \mathrm{J}=12.4 \mathrm{~Hz}, 3.2 \mathrm{~Hz}\right.$ ), $3.53-3.81\left(3 \mathrm{H}, \mathrm{m}, \mathrm{CH}_{2} \mathrm{OH}\right.$, 
6- $\left.\mathrm{H}_{\mathrm{b}}\right), 3.67$ and $3.69\left(3 \mathrm{H}, \mathrm{s}, \mathrm{OCH}_{3}\right), 4.10-4.35(1 \mathrm{H}, \mathrm{m}, 5-\mathrm{H}) .-{ }^{13} \mathrm{C}-\mathrm{NMR}\left(\mathrm{CDCl}_{3}\right): \delta(\mathrm{ppm})$ $=-5.1$ and $-4.8\left(\mathrm{SiCH}_{3}\right), 17.9$ (Me $\mathrm{MeSi}_{3}, 25.7$ (Mes $\left.\mathrm{MSS}_{3}\right), 19.0$ and 19.4 (C-3), 27.4 and 29.7 (C-4), $46.4(\mathrm{C}-2), 51.7$ (C-6), 52.5 and $52.7\left(\mathrm{OCH}_{3}\right), 60.3,60.6$, and $61.1\left(\mathrm{CH}_{2} \mathrm{OH}\right)$ (rotamers), 64.1, $67.6(C-5), 157.7$ and $157.9(C=0)$.

\section{(2S,5s)-5-t-Butyldimethylailyloxy-N-methoxycarbony-2-hydroxymethyl-piperidine (11a)}

A diasterecumeric mixture of $11 \mathrm{a}, \mathrm{b}(2.8 \mathrm{~g}, 9.25 \mathrm{mmol})$ was chromatographed on silica gel (500 9. column diameter $5 \mathrm{~cm}$ ) using ethyl acetat. Eight $50 \mathrm{ml}$ fractions were collected, the solvents evaporated and the diastereomeric purity of the oily residues were checked by $13 \mathrm{C}$. NMR spectrocopy. The first 5 fractions contained $1.54 \mathrm{~g} 11 \mathrm{a}$ (cis), $1.14 \mathrm{~g}$ was unseparated cis/trans mixture.- ${ }^{1} \mathrm{H}$-NMR ( $\left.\left.\mathrm{CDCl}_{3}\right): 0.03(6 \mathrm{H}, \mathrm{s}, \mathrm{SiMe})_{2}\right), 0.83(9 \mathrm{H}, \mathrm{s}$, tBuSi), 1.43 - 1.78 (4 $H, m, 3-H, 4-H), 2.56-2.81$ (1 H, broad, OH), $3.51-3.67(3 \mathrm{H}, \mathrm{m}, 2-H, 6-H), 3.69(3 H, s$, $\left.\mathrm{OCH}_{3}\right) 3.79(2 \mathrm{H}$, dd, $J=11.1 \mathrm{~Hz}, 8.8 \mathrm{~Hz}), 4.10-4.35(1 \mathrm{H}, \mathrm{m}, 5-\mathrm{H}) .-13 \mathrm{C}-\mathrm{NMR}\left(\mathrm{CDCl}_{3}\right): \delta$ (ppm) $=-4.8$ ( $\left.\mathrm{SiCH}_{3}\right), 18.0$ (Me3 $\mathrm{CSi}$ ), 22.7 (C-3), 25.7 (Me3CSi), 29.7 (C-4), 46.5 (C-2), $51.7(\mathrm{C}-6), 52.7\left(\mathrm{OCH}_{3}\right), 60.6,61.1\left(\mathrm{CH}_{2} \mathrm{OH}\right)$ (rotamers), $67.6(\mathrm{C}-5), 157.0(\mathrm{C}=0)$.

\section{(2R,S-5S)-t-Butyldimethylalyloxy-N-methoxycarbony-plperidine-2-carboxaldehyde (12a,b)}

To a cooled $\left(-60^{\circ} \mathrm{C}\right.$.) solution of oxalyl chloride $(0.36 \mathrm{ml}, 4.05 \mathrm{mmol})$ in dry dichloromethane $\left(10 \mathrm{ml}\right.$ ) dimethylsulfoxide $(0.58 \mathrm{ml}, 8.1 \mathrm{mmol})$ in dry $\mathrm{CH}_{2} \mathrm{Cl}_{2}(10 \mathrm{ml})$ was added dropwise so that the temperature did not rise above $-50{ }^{\circ} \mathrm{C}$. $11 \mathrm{a}, \mathrm{b}(1.12 \mathrm{~g}, 3.67 \mathrm{mmol})$ was dissolved in dry $\mathrm{CH}_{2} \mathrm{Cl}_{2}(10 \mathrm{ml})$ and was added within $5 \mathrm{~min}$. The solution was stirred for another $15 \mathrm{~min} .$, then triethylamine $(2.55 \mathrm{ml}, 18.4 \mathrm{mmol})$ was added, and the mixture was stirred for $5 \mathrm{~min}$. After the mixture was allowed to warm to ambient temp. water $(50 \mathrm{ml})$ was added and the layers were separated. The aqueous layer was extracted with $\mathrm{CH}_{2} \mathrm{Cl}_{2}(2 \times 80 \mathrm{ml})$. The combined organic layers were washed with saturated sodium chloride solution (120 mi) and then dried with sodium sulphate. After filtration and evaporation of the solvent, the oil was purified by flash chromatography. Yield: $0.78 \mathrm{~g}(72 \%)$ colorless oil as a mixture of diastereomers in a ratio of 7:3. I):diastereomer $30 \%$; ${ }^{\prime 1}$ : :diastereomer $70 \% .-R_{f}=0.72 \mathrm{~g}$ (petroleum ether/ EtOAc 2:1).- IR (neat): $2985,2940,2860$ (CH), 1745 (HC=0), 1715 $(\mathrm{NC}=0), 1255(\mathrm{C}-0) \mathrm{cm}^{-1}$.- ${ }^{1} \mathrm{H}-\mathrm{NMR}\left(\mathrm{CDCl}_{3}\right): \delta(\mathrm{ppm})=0.05\left(6 \mathrm{H}, \mathrm{d}, \operatorname{SiM} \theta_{2}\right), 0.86(9 \mathrm{H}, \mathrm{s}$, tBuSi), $\left.1.21-2.70\left(5 \mathrm{H}, \mathrm{m}, 3-\mathrm{H}, 4-\mathrm{H}, 6-\mathrm{H}_{\mathrm{a}}\right), 3.00\left(1 \mathrm{H}, \mathrm{d}, 6-\mathrm{H}_{\mathrm{b}}{ }^{1}\right), \mathrm{J}=14.6 \mathrm{~Hz}\right), 3.55-3.98$ (2 $\left.\mathrm{H}, \mathrm{m}, 2-\mathrm{H}, 6-\mathrm{H}_{\mathrm{b}}^{\prime \prime \prime}, 3.71^{\prime \prime}\right)$ and $\left.3.75^{\prime \prime}\left(3 \mathrm{H}, \mathrm{s}, \mathrm{OCH}_{3}\right), 4.58-4.67(1 \mathrm{H}, \mathrm{m}, 5-\mathrm{H}), 9.59^{\prime \prime}\right)$ (1 $H, s, H C=0), 9.60^{\prime \prime}(1 \mathrm{H}, d, J=0.7 \mathrm{~Hz}, \mathrm{HC}=0)$. $\left.{ }^{13} \mathrm{C}-\mathrm{NMR}\left(\mathrm{CDCl}_{3}\right): \delta(\mathrm{ppm})=-5.0^{\prime \prime}\right)$ and -

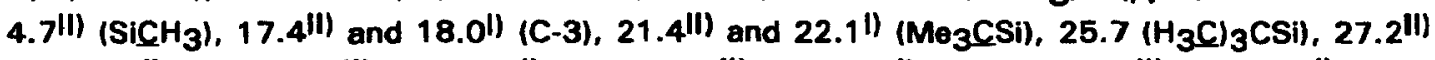

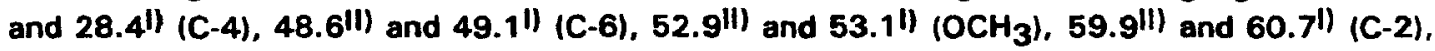

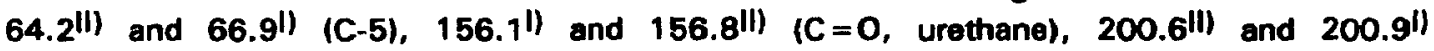
$\left(\mathrm{HC}=0\right.$ ).- $\mathrm{C}_{14} \mathrm{H}_{27} \mathrm{NO}_{4} \mathrm{Si}$ (301.41).- Calcd.: C 55.77 H 9.02 N 4.60 found: C 55.82 H $9.29 \mathrm{~N}$ 4.61. 
Methyl (2S,5s)-5-t-Butyldimethylailyloxy-N-methoxycarbony-plperidine-2-carboxylate (13a)

$11 \mathrm{a}(1.64 \mathrm{~g}, 5.4 \mathrm{mmol})$ was oxidized to the aldehyd under Swern conditions as described for 12a,b. The resulting brown oil was dissolved in methanol/water 9:1 (30 $\mathrm{ml}$ ) then solid $\mathrm{NaHCO}_{3}(9.1 \mathrm{~g}, 108 \mathrm{mmol})$ and bromine $(0.55 \mathrm{ml}, 16.9 \mathrm{mmol})$ was added. The mixture was stirred for $1.5 \mathrm{~h}$ at $25^{\circ} \mathrm{C}$. The excess of bromine was reduced with sodium thiosulphate and the solution was filtered. The solvent was evaporated and the residue was stirred in $80 \mathrm{ml}$ of ether. Filtration over a short column of silicagel (petroleum ether/EtOAc 2:1) and evaporation of the solvent provided a nearly colourless oil. Yield: $1.42 \mathrm{~g}(79 \%$ ). Rf: 0.86 (petroleum ether/EtOAC 2:1).- $[\alpha]_{D}^{20}=-15.4(C=1, \mathrm{MeOH})$.- IR (neat): 2960, 2925, 2895, 2860 (CH), $1750\left(\mathrm{C}=\mathrm{O}\right.$ ester), $1710\left(\mathrm{C}=0\right.$, urethane), $1255(\mathrm{C}-\mathrm{O}) \mathrm{cm}^{-1}$.- ${ }^{1} \mathrm{H}-\mathrm{NMR}\left(\mathrm{CDCl}_{3}\right): \delta(\mathrm{ppm})=$ 0.06 - 0.05 (6 H, m, SiMe 2 rotamers), 0.86 (9 H, s, tBuSi), 1.32 - 2.31 (5 H, m, 3-H, 4-Hab. $\mathrm{OH}), 2.65-2.75\left(1 \mathrm{H}, \mathrm{m}, 6-\mathrm{H}_{\mathrm{a}}\right) 3.49-3.66\left(1 \mathrm{H}, \mathrm{m}, 6-\mathrm{H}_{\mathrm{b}}\right), 3.69\left(3 \mathrm{H}, \mathrm{s}, \mathrm{OCH}_{3}\right), 3.72(3 \mathrm{H}$, $\left.\mathrm{s}, \mathrm{OCH}_{3}\right), 3.87-4.18(1 \mathrm{H}, \mathrm{m}, 5-\mathrm{H}), 4.71$ - 4.86 (1 H, m, 2-H, rotamers). ${ }^{13} \mathrm{C}-\mathrm{NMR}\left(\mathrm{CDCl}_{3}\right)$ : $\left.\delta(\mathrm{ppm})=-4.8\left(\mathrm{SiCH}_{3}\right), 18.0\left(\mathrm{Me}_{3} \mathrm{CSi}\right), 24.7(9), 25.3(\mathrm{C}-3) 26.0\left(\mathrm{CH}_{3}\right)_{3} \mathrm{CSi}\right) 31.0$ (C-4), $48.2,48.4$ (rotamers) (C-6), 52.2, $\left.\mathrm{COCH}_{3}\right)$ 53.3, 53.6 (rotamers) (C-2), 67.2, 67.9 (rotamers) (C-5), $156.6(C=0$, urethane), $171.5(C=0$, ester).

\section{Methyl (25,5S1-Hydroxy-N-methoxycarbonyl-piperidine-2-carboxylate (14a)}

$13 \mathrm{a}(0.72 \mathrm{~g}, 2.17 \mathrm{mmol})$ was dissolved in ether $(40 \mathrm{ml})$ and cooled to $0^{\circ} \mathrm{C}$. Then dry methanolic hydrogen chloride $(10 \mathrm{ml})$ was added and stored $2 \mathrm{~d}$ at $4{ }^{\circ} \mathrm{C}$. The solvent and TBDMSOCH $\mathrm{C}_{3}$ was evaporated and the resulting oil was purified by flash chromatography over silica gel (EtOAc). Yield: $0.33 \mathrm{~g}(70 \%)$ - $\mathbf{R}_{f}=0.42$ (EtOAC).- $[\alpha]_{\mathrm{D}}^{20}=-24.1(\mathrm{c}=1$, MeOH).- IR (neat): $3460(\mathrm{OH}), 3010,2960,2880(\mathrm{CH}), 1745(\mathrm{C}=0), 1710,1690$ $(\mathrm{C}=\mathrm{O}) \mathrm{cm}{ }^{1}$ - $\mathrm{H}-\mathrm{NMR}\left(\mathrm{CDCl}_{3}\right): \delta(\mathrm{ppm})=1.20-1.31(1 \mathrm{H}, \mathrm{m}, 3-\mathrm{H}), 1.70-1.93(3 \mathrm{H}, \mathrm{m}$, 4-H, 3-H OH), $2.25-2.33(1 \mathrm{H}, \mathrm{m}, 4-\mathrm{H}), 2.74-2.82\left(1 \mathrm{H}, \mathrm{m}, 6-\mathrm{H}_{\mathrm{a}}\right), 3.57-3.65(1 \mathrm{H}, \mathrm{m}$, $\left.6-H_{b}\right), 3.72\left(3 H, s, O C H_{3}\right), 3.74\left(3 H, s, C^{2 O O C H}\right)_{3}, 4.05-4.25(1 H, m, 5-H), 4.71-$ $4.93(1 \mathrm{H}, \mathrm{m}, 2-\mathrm{H})$, signals are broadened by rotamers.- ${ }^{13} \mathrm{C}-\mathrm{NMR}\left(\mathrm{CDCl}_{3}\right): 8(\mathrm{ppm})=$ 24.9 (C-3), $30.3(\mathrm{C}-4), 48.0(\mathrm{C}-6), 52.3\left(\mathrm{OCH}_{3}\right), 53.0\left(\mathrm{OCH}_{3}\right), 53.3(\mathrm{C}-2), 66.5$ (C-5), $157.3\left(\mathrm{C}=0\right.$, urethane), $171.5\left(\mathrm{C}=0\right.$, ester). $\mathrm{C}_{9} \mathrm{H}_{15} \mathrm{NO}_{5}$ (217.22).- Calcd.: $\mathrm{C} 49.76 \mathrm{H}$ 6.96 N 6.44 found: C 48.93 H 7.58 N 5.72.

\section{Methyl (2R,5S)-5-Hydroxy-N-methoxycarbonyl-plperldine-2-carboxylate (14b)}

From $660 \mathrm{mg}(2.08 \mathrm{mmal}) 13 \mathrm{~b}$, as described for $14 \mathrm{a}$. Yield: $390 \mathrm{mg}$ - $(86 \%)$.- $R_{f}=0.40$ (EtOAc).- IR(neat): 3460 (OH), 3020-2880, 1740 (C=0 ester), 1710-1685 (C =0 urethane), 1450, 1405, 1370, $\mathrm{cm}^{-1} .{ }^{1} \mathrm{H}-\mathrm{NMR}\left(\mathrm{CDCl}_{2}\right)\left(60^{\circ} \mathrm{C}\right): \delta(\mathrm{ppm})=1.30-2.30(5 \mathrm{H}$ , $\mathrm{m}, 3-\mathrm{H}, 4-\mathrm{H}, \mathrm{OH}), 3.22\left(1 \mathrm{H}, \mathrm{d}, \mathrm{J}_{6 a b}=14.1 \mathrm{~Hz}_{2}, 6-\mathrm{H}_{\mathrm{a}}\right), 3.56-4.25\left(8 \mathrm{H}, \mathrm{m}, 6-\mathrm{H}_{\mathrm{b}}, \mathrm{H}-5\right.$, $\mathrm{OCH}_{3}$ ester + $\mathrm{OCH}_{3}$ urethane), $4.88(1 \mathrm{H}-\mathrm{m}, 2-\mathrm{H}) .-{ }^{13} \mathrm{C}-\mathrm{NMR}\left(\mathrm{CDCl}_{3}\right): \delta(\mathrm{ppm})=19.5$ 
(C-3), 26.5 (C-4), 46.6 (C-6), 51.9, $\left(\mathrm{OCH}_{3}\right) 52.6\left(\mathrm{OCH}_{3}\right) 52.9$ (C-2), 62.8 (C-5), 156.4 $(C=0$, urethane), $170.1(C=0$, ester $)$.

\section{(2S,5S)-5-Hydroxy-piperidine-2-carboxylic acid hydrochloride (15a)}

14a $(0.49 \mathrm{~g}, 2.26 \mathrm{mmol})$ was refluxed $4 \mathrm{~h}$ in $6 \mathrm{M} \mathrm{HCl}(10 \mathrm{ml})$. After concentration in vacuo $\mathrm{EtOH} / \mathrm{Et}_{2} \mathrm{O}$ was added to the oily residue. The solvent was evaporated and the residue was dissolved in EtOH/THF $(20 \mathrm{ml})$ and refluxed after addition of a small amount of charcoal. After filtration and evaporation of the solvent, the cristalline solid was triturated with acetone/EtOH (1:1) and isolated by suction. Yield: $0.31 \mathrm{~g}\left(76 \%\right.$ ).- m.p.: $195-196^{\circ} \mathrm{C}$ (dec.), \{ref. 19 183$\left.187^{\circ} \mathrm{C}\right)$ - $[\alpha]_{\mathrm{D}}^{20}=-21.9\left(\mathrm{c}=1, \mathrm{H}_{2} \mathrm{O}\right)$, (ref. $\left.{ }^{19}-18.5\left(c=1.0, \mathrm{H}_{2} \mathrm{O}\right)\right)_{\text {. }}-\mathrm{IR}(\mathrm{KBr}): 3480,3390$ $(\mathrm{OH}), 3150-2400\left(\mathrm{CH}, \mathrm{NH}_{2}{ }^{+}\right), 1735(\mathrm{C}=0), 1555 \mathrm{~cm}^{-1}$.- ${ }^{1} \mathrm{H}-\mathrm{NMR}\left(\mathrm{D}_{4}-\mathrm{MeOD}\right): \delta(\mathrm{ppm})=$ $1.83-2.19(4 H, m, 3-H, 4-H), 3.22-3.26(2 H, m, 6-H), 3.99(1 H, t, J=7.4 H z, 2-H), 4.07-$ $4.1(1 \mathrm{H}, \mathrm{m}, 5-\mathrm{H}) .-{ }^{13} \mathrm{C}-\mathrm{NM}$ (D $\left.\mathrm{D}_{4}-\mathrm{MeOD}\right): \delta(\mathrm{ppm})=21.9(\mathrm{C}-3), 29.8(\mathrm{C}-4), 50.4(\mathrm{C}-6), 57.6$ (C-2), 62.1 (C-5), 170.6 (COOD). $\mathrm{C}_{6} \mathrm{H}_{12} \mathrm{CINO}_{3}$ (181.62) Calcd.: C $39.68 \mathrm{H} 6.66 \mathrm{~N} 7.71$ found: $\mathrm{C} 39.88 \mathrm{H} 7.09 \mathrm{~N} 7.57$.

\section{(2R,5S)-5-Hydroxy-piperidine-2-carboxylic acid hydrochloride (15b)}

$14 \mathrm{~b}(217 \mathrm{mg}, 1.00 \mathrm{mmol})$ was refluxed for $4 \mathrm{~h}$ with $6 \mathrm{M} \mathrm{HCl}(2 \mathrm{ml})$. After evaporation of the volatilas the residue was triturated with EtOH/acetone. The crystalline solid was isolated by vacuum filtration. Yield: $130 \mathrm{mg}(72 \%)$.- m.p.: 225 (dec.).- $[\alpha]_{1}^{20}=+8.6\left(c=1, \mathrm{H}_{2} \mathrm{O}\right)\langle$ ref. 19 for 2S, 5R: - $9.7\left(\mathrm{c}=0.9, \mathrm{H}_{2} \mathrm{O}\right)$ ). - IR $(\mathrm{KBr})$ : $3360,3220(\mathrm{OH}), 3020-2420\left(\mathrm{CH}, \mathrm{NH}_{2}+\right), 1745$ $(C=0), 1590 \mathrm{~cm}^{-1},{ }^{1} \mathrm{H}-\mathrm{NMR}\left(\mathrm{D}_{4}-\mathrm{MeOD}\right): \delta(\mathrm{ppm})=1.65\left(1 \mathrm{H}, \mathrm{m}, 4-\mathrm{H}_{\mathrm{a}}\right), 1.86\left(1 \mathrm{H}, \mathrm{m}, 3-\mathrm{H}_{\mathrm{a}}\right)$, $2.02\left(1 \mathrm{H}, \mathrm{m}, 4-\mathrm{H}_{b}\right), 2.39\left(1 \mathrm{H}, \mathrm{m}, 3-\mathrm{H}_{b}\right), 2.85\left(1 \mathrm{H}, \mathrm{dd}, \mathrm{J}_{\mathrm{ab}}=12.2,9.2 \mathrm{~Hz}, 6-\mathrm{H}_{\mathrm{a}}\right), 3.42(1 \mathrm{H}$, ddd, $\left.J=12.2,3.9,1.3 \mathrm{~Hz}, 6-\mathrm{H}_{b}\right), 3.90(1 \mathrm{H}, \mathrm{m}, 5-\mathrm{H}), 4.01(1 \mathrm{H}, \mathrm{dd}, J=10.1,3.9 \mathrm{~Hz}, 2-\mathrm{H})$.${ }_{13} \mathrm{C}-\mathrm{NMR}\left(\mathrm{D}_{4}-\mathrm{MeOD}\right): \delta(\mathrm{ppm})=24.4$ (C-3), 31.1 (C-4), 49.1 (C-6), 56.9 (C-2), 63.9 (C-5), $170.7(\mathrm{C}=0)$. $-\mathrm{C}_{6} \mathrm{H}_{12} \mathrm{CINO}_{3}(181.62)$ Calcd.: $\mathrm{C} 39.68 \mathrm{H} 6.66 \mathrm{~N} 7.71$ found: $\mathrm{C} 39.46 \mathrm{H} 6.64$ N 7.62 .

Methyl (2R,5R)-5-benzoyloxy-N-methoxycarbonyl-piperidine-2-carboxylate (17) $80 \%$ d.e. $300 \mathrm{mg}(1.38 \mathrm{mmol}) 16$ (prepared in $80 \%$ d.e. as described in ref. $1 \mathrm{~g}$ ), triphenylphosphine $(472 \mathrm{mg}, 1.80 \mathrm{mmol}$ ) and benzoic acid (220 $\mathrm{mg}, 1.80 \mathrm{mmol}$ ) were dissolved in THF $(20 \mathrm{ml})$ under $\mathrm{N}_{2}$. To this mixture was added with stirring diethyl azodicarboxylate $(313 \mathrm{mg}, 1.80 \mathrm{mmol})$ in THF $(2 \mathrm{ml})$. After $1 \mathrm{~h}$ the solvent was evaporated in vacuo and $\mathrm{CH}_{2} \mathrm{Cl}_{2}(100 \mathrm{ml})$ was added. The mixture was washed with 5 $\% \mathrm{NaHCO}_{3}$ solution $(2 x)$ and water $(1 x)$. The organic layer was dried $\left(\mathrm{Na}_{2} \mathrm{SO}_{4}\right)$ and evaporated. The residue was chromatographed on silica gel $\left(\mathrm{CHCl}_{3} / \mathrm{EtOAc} 9+1\right)$. Yield: $189 \mathrm{mg}(43 \%)$ colorless oil $80 \%$ d.e. by ${ }^{1} \mathrm{H}-\mathrm{NMR}$. $\mathbf{R}_{f}=0.40\left(\mathrm{CHCl}_{3} / \mathrm{EtOAC} 9+1\right)$. IR 
(neat): $3070-2880,1750-1690(\mathrm{C}=0), 1605,1585 \mathrm{~cm}^{-1} .{ }^{1} \mathrm{H}-\mathrm{NMR}\left(\mathrm{CDCl}_{3}\right)$ at $60^{\circ} \mathrm{C}$; only signals for $2 R, 5 R 17$ are shown: $\delta(p p m)=1.62\left(1 \mathrm{H}, d q, J_{3 a, 4 a} \approx J_{4 a, 4 a} \approx\right.$ $\left.J_{4 a, 5 a} \approx 11-14 \mathrm{~Hz}, J_{3 e, 4 a}=3.8 \mathrm{~Hz}, 4-\mathrm{H}_{a}\right) .1 .89\left(1 \mathrm{H}, d d t, J_{3 a, 4 a}=J_{3 a, 3 e}=13.9 \mathrm{~Hz}\right.$, $\left.J_{3 a, 2 \theta}=6.1 \mathrm{~Hz}, J_{3 a, 4 \theta}=3.5 \mathrm{~Hz}, 3-\mathrm{H}_{a}\right), 2.17\left(1 \mathrm{H}, \mathrm{m}, 4-\mathrm{H}_{\theta}\right), 2.36\left(1 \mathrm{H}\right.$, ddd, $\mathrm{J}_{3 a, 3 \theta}=$ $\left.13.9 \mathrm{~Hz}, J_{2 e, 3 e}=6.2 \mathrm{~Hz}, J_{3 e, 4 a}=3.8 \mathrm{~Hz}, 3-\mathrm{H}_{\theta}\right), 3.08\left(1 \mathrm{H}, d d, J_{6 a, 6 e}=12.4 \mathrm{~Hz}\right.$, $\left.J_{5 a, 6 a}=10.9 \mathrm{~Hz}, 6-\mathrm{H}_{\mathrm{a}}\right), 3.74\left(3 \mathrm{H}, \mathrm{s}, \mathrm{OCH}_{3}\right), 3.77\left(3 \mathrm{H}, \mathrm{s}, \mathrm{OCH}_{3}\right), 4.38\left(1 \mathrm{H}, \mathrm{dd}, \mathrm{J}_{6 \mathrm{a}}, 6 \theta\right.$ $\left.=12.4 \mathrm{~Hz}, \mathrm{~J}_{5 \mathrm{a}, 6 \mathrm{\theta}}=5.0 \mathrm{~Hz}, 6-\mathrm{H}_{\theta}\right), 4.89-5.04\left(2 \mathrm{H}, \mathrm{m}, 2-\mathrm{H}_{\theta}, 5-\mathrm{H}_{\mathrm{a}}\right), 7.37-7.59(3 \mathrm{H}, \mathrm{m}$, $\mathrm{H}_{\text {arom }}$, 7.98-8.04 (2H, m, $\mathrm{H}_{\text {arom }}$ ) - ${ }^{13} \mathrm{C}-\mathrm{NMR}\left(\mathrm{CDCl}_{3}\right): 24.60 / 24.93$ (C-4), 26.34/26.84 (C-3), 44.60/44.79 (C-6), 52.34/53.07 (2x $\left.\mathrm{OCH}_{3}\right), 53.27 / 53.58$ (C-2), 68.65 (C-5), 128.28, 129.52, $129.79,133.09$ ( $\mathrm{C}_{\text {arom }}$ ), 156.02/156.49 $\left(\mathrm{CO}_{2} \mathrm{CH}_{3}\right.$ urethane), 165.52 $\left(\mathrm{PhCO}_{2}\right.$-), $171.30\left(\mathrm{CO}_{2} \mathrm{CH}_{3}\right.$ ester)-rotamers.- $\mathrm{C}_{16} \mathrm{H}_{1} \mathrm{NNO}_{6}$ (321.33) Calcd.: C $59.81 \mathrm{H}$ 5.96 N 4.36 found.: C 59.78 H 5.91 N 4.2O.

(2R,5R)-5-Hydroxy-piperidine-2-carboxylic acid hydrochloride (18) 84\% d.e.

$17(80 \%$ d.e.) (175 mg, $545 \mu \mathrm{mol})$ was refluxed with $4 \mathrm{M} \mathrm{HCl}(3 \mathrm{ml})$ for $6 \mathrm{~h}$. After cooling, benzoic acid was extracted from the aqueous phase with $\mathrm{CH}_{2} \mathrm{Cl}_{2}(3 x)$. After evaporation of the aqueous layer the oily residue crystallized. The colorless crystals were triturated with acetone, isolated by vacuum filtration and dried. Yield: $58 \mathrm{mg}$ (59\%).m.p.: $187^{\circ} \mathrm{C}-195^{\circ} \mathrm{C}$ (dec.). $[\alpha]_{D}^{20}=+18.1(\mathrm{c}=1.3 \mathrm{MeOH})\left(84 \%\right.$ d.e. by ${ }^{1} \mathrm{H}-\mathrm{NMR}$ ). - IR (KBr): 3520, $3490(\mathrm{OH}), 3000-2500\left(\mathrm{CH}_{,} \mathrm{NH}_{2}{ }^{+}\right), 1730(\mathrm{C}=0), 1560,1440,1400$, 1225, ${ }^{1} \mathrm{H}-\mathrm{NMR}\left(\mathrm{D}_{4}-\mathrm{MeOD}\right): \delta(\mathrm{ppm})=1.83-2.24(4 \mathrm{H}, \mathrm{m}, 3-\mathrm{H}, 4-\mathrm{H}), 3.23(2 \mathrm{H}, \mathrm{m}, \mathrm{H}-6)$, $4.02(1 \mathrm{H}, t, J=7.5 \mathrm{~Hz}, 2-\mathrm{H}), 4.08-4.13(1 \mathrm{H}, \mathrm{m}, 5-\mathrm{H}) .-{ }^{13} \mathrm{C}-\mathrm{NMR}\left(\mathrm{D}_{4}-\mathrm{MeOD}\right): \delta(\mathrm{ppm})=$ 21.8 (C-3), 29.8 (C-4), 50.3 (C-6), 57.6 (C-2), 62.1 (C-5), 170.7 (COOD). $\mathrm{C}_{6} \mathrm{H}_{12} \mathrm{CINO}_{3}$ (181.62) Calcd.: C 39.68 H 6.66 N 7.71 found: C 39.79 H 6.54 N 7.66.

\section{References and Notes}

1. a. Pipecolic Acids II : C. Herdeis and W. Engel, Arch. Pharm. (Weinheim), 1992, 325, 411 417. b. P. D. Bailey, G. R. Brown, F. Korber, A. Reed and R. D. Wilson, Tetrahedron: Asymmetry, 1991, 2, 1263-1282. c. P. D. Bailey and J. S. Bryans, Tetrahedron Lett. 1988, 29, 2231-2234. d. Leading references for the syntheses of subst. pipecolic acids: S. R. Angle and D. O. Arnaiz, Tetrahedron Lett. 1989, 30, 515-518, see ref. 3 and 4. e. W. N. Speckamp, H. Hiemstra and P. M. Esch, Tetrahedron Lett. 1990, 31, 759-762. f. H. Rapoport and R. C. Roemmele, J. Org. Chem. 1989, 54, 1866-1875. g. C. Herdeis and W. Engel, Tetrahedron: Asymmetry, 1991, 2, 945-948. h. F. Couty and C. Agami, Synlett, 1990, 731-732.

2. a. J. P. Whitten, R. v. Cube, B. M. Baron and I. A. Mc Donald, BioMed. Chem. Lett. 1993 , 3, 19-22. b. T. D. Copeland, E. M. Wondrak, J. Toszer, M. M. Roberts and S. Oroszlan, Biochem. Biophys. Res. Comm. 1990, 169, 310-314. 
3. C. Angst, P. S. Bernard, L. Blanchard, T. Campbell, R. De Josus, W. Guida, A, J. Hutchison, R. H. Jackson, D. E. Murphy, M. A. Stills, M. Williams and E. Wilusz, J. Med. Chem. 1989, 32, 2171-2178.

4. P. L. Omstein, D. D. Schoepp, M. B. Arnold, J. D. Leander, D. Lodge, J. W. Paschal and T. Elzey, J. Med. Chem. 1991, 34, 90-97.

5. C. Herdeis and W. Held, unpublished.

6. Synthesis of this amino acid is not known. Isolation as a diketopiperazine derivative: $\mathbf{G}$. $\mathbf{R}$. Pettit, R. B. Von Dreele, D. L. Herald, M. T. Edgar and H. B. Wood, Jr., J. Am. Chem. Soc. 1976, 98, 6742-6743. total synthesis: T. Fukuyama, R. K. Frank and C. F. Jewell, Jr., J. Am. Chem. Soc. 1980, 102, $2122-2123$.

7. Syntheais with: G. J. Hanson and M. A. Russel, Tetrahedron Lett. 1989, 30, 5751-5754. J. P. Mazaleyrat, I. Rage, J. Xie, J. Sayrada and M. Wakselman, Tetrahedron Lett. 1992, 33, 4453-4456. Synthesis of: C. Herdeis and W. Engel, Arch. Pharm. (Woinheim) 1993, 326, 297-301. A. W. Burgstahler and C. E. Aiman, J. Org. Chem. 1960, 25, 489-492. N. A. Dobson and R. A. Raphael, J. Chem. Soc. 1958, 3642-3647.

8. a. F. N. Tebbe, G. W. Parshall and G. S. Reddy, J. Am. Chem. Soc. 1978, 100, 3611 3613. b. R. H. Grubbs and L. F. Cannizzo, J. Org. Chem. 1985, 50, 2316-2323. c. S. H. Pine, R. J. Pettit, G. D. Geib, S. G. Cruz, C. H. Gallego, T. Tijerina and R. D. Pine, J. Org. Chem. 1985, 50, 1212-1216. d. S. H. Pine, G. S. Shen and H. Hoang, Synthesis 1991, 165-167. Review: S. H. Pine, Org. React. 1993, 43, 1-91.

9. a. K. Oshima, H. Nozaki, K. Takai, Y. Hotta, Tetrahedron Lett. 1978, 27, 2417-2420. b. L. Lombardo, Tetrahedron Lett. 1982, 23, 4293-4296.

10. K. Clauss and H. Bestian, Liebigs Ann. Chem. 1962, 654, 8-19.

11. N. A. Petasis and E. I. Bzowej, J. Am. Chem. Soc. 1990, 112, 6392-6394.

12. Benzylidenations of dimethyl formamide and dimethyl acetamide with Dibenzyltitanocene is described: N. A. Petasis and E. I. Bzowej, J. Org. Chem. 1992, 57, 1327-1330.

13. 2-Pyrrolidone and 2-azetidinone derivatives are also methylenated in $50-60 \%$ yield. Also 2 piperidone $\mathrm{i}^{14}$ is transformed to $\mathrm{ii}$.

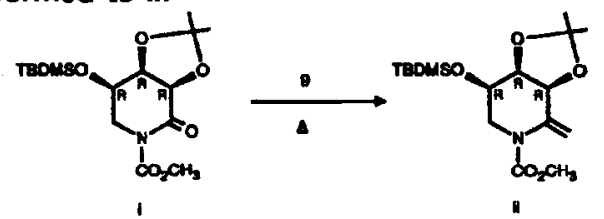

14. C. Herdeis and D. Waibel, Arch. Pharm. (Weinheim), 1991, 324, 269-274.

15. 10 is a rather stable compound. It can be distilled in vacuo and treated with $3 \mathrm{M} \mathrm{NaOH}$ solution at r.t. without decomposition. Flash chromatography on silica gel must be performed with some drops of EtzN in the eluent.

16. K. B. Sharpless, P. H. J. Carlsen, T. Katsuki and V. S. Martin, J. Org. Chem. 1981, 46. 3936-3938.

17. F. W. Lichtenthaler, P. Jarglis and K. Lorenz, Synthesis 1988, 790-792.

18. C. Herdeis, Synthesis 1986, 232-233.

19. S. -1. Hatanaka, S. Kaneko, Phytochemistry, 1977, 16, 1041-1042. 